\title{
THE TOP-DOG INDEX: A NEW MEASUREMENT FOR THE DEMAND CONSISTENCY OF THE SIZE DISTRIBUTION IN PRE-PACK ORDERS FOR A FASHION DISCOUNTER WITH MANY SMALL BRANCHES
}

\author{
SASCHA KURZ, JÖRG RAMBAU, JÖRG SCHLÜCHTERMANN, AND RAINER WOLF
}

\begin{abstract}
We propose the new Top-Dog-Index, a measure for the branch-dependent historic deviation of the supply data of apparel sizes from the sales data of a fashion discounter. A common approach is to estimate demand for sizes directly from the sales data. This approach may yield information for the demand for sizes if aggregated over all branches and products. However, as we will show in a real-world business case, this direct approach is in general not capable to provide information about each branch's individual demand for sizes: the supply per branch is so small that either the number of sales is statistically too small for a good estimate (early measurement) or there will be too much unsatisfied demand neglected in the sales data (late measurement). Moreover, in our real-world data we could not verify any of the demand distribution assumptions suggested in the literature. Our approach cannot estimate the demand for sizes directly. It can, however, individually measure for each branch the scarcest and the amplest sizes, aggregated over all products. This measurement can iteratively be used to adapt the size distributions in the pre-pack orders for the future. A real-world blind study shows the potential of this distribution free heuristic optimization approach: The gross yield measured in percent of gross value was almost one percentage point higher in the test-group branches than in the control-group branches.
\end{abstract}

\section{INTRODUCTION}

The financial performance of a fashion discounter depends very much on its ability to predict the customers' demand for individual products. More specifically: trade exactly what you can sell to your customers. This task has two aspects: offer what your customers would like to wear because the product as such is attractive to them and offer what your customers can wear because it has the right size.

In this paper, we deal with the second aspect only: meet the demand for sizes as accurately as possible. The first aspect, demand for products, is a very delicate issue: Products in a fashion discounter are never replenished because of lead times of around three months. Therefore, there will never be historic sales data of an item at the time when the order has to be submitted (except for the very few "never-out-of-stock"-items, NOS items, for short).

When one considers the knowledge and experience of the professional buyers employed at a fashion discounter - acquired by visiting expositions, reading trade journals, and the like - it seems hard to imagine that a forecast for the demand for a product could be implemented in an automated decision support system at all. We seriously doubt that the success of fashion product can be assessed by looking at historic sales data only. In contrast to this, the demand for sizes may stay reasonably stable over time to extract useful information from historic sales data.

2000 Mathematics Subject Classification. Primary: 90B05; Secondary: 90B90.

Key words and phrases. revenue management, size optimization, demand forecasting, Top-Dog-Index, field study, parallel blind testing.

This research was supported by the Bayerische Forschungsstiftung, Project DISPO_A Decision Support for the Integrated Size and Price Optimization. 
In the historic sales data the influences of demand for products and demand for sizes obviously interfere. Moreover, it was observed at our partners' branches that the demand for sizes seems not to be constant over all around 1200 branches.

The main question of this work is: how can we forecast the demand for sizes individually for each branch or for a class of branches?

1.1. Related Work. Interestingly enough, we have not found much work that exactly deals with our task. It seems that, at first glance, the problem of determining the size distribution in delivery pre-packs can be considered as simple regression once you have historic sales data: Just estimate the historic size profile and fit your delivery to that. At least two trivial US-patents 6. 8] have recently been granted and published along these lines (which witnesses that the US patent system may not have employed the necessary expertise in their patent evaluations ...).

In our problem, however, the historic sales data is not necessarily equal to the historic demand data, and it is interesting how to find the demand data in the sales data in the presence of unsatisfied demand and very small delivery volumes per branch and per product.

The type of research closest to ours seems to be classified as assortment optimization. In a sense, we want to decide on the start inventory level of sizes in a pre-pack for an individual product in an individual branch. (Let us ignore for a moment that these unaggregated inventory levels are very small compared to other inventory levels, e.g., for grocery items.)

Mostly, the successful approaches deal rather with NOS items than with perishable and not replenishable fashion goods. For example, assortment optimization in the grocery sector 4, Section 4] - one of the very few papers documenting a field study - can usually neglect the effects of stockout substitution in sales data, which make demand estimation from sales data much more reliable. There is work on the specific influence of substitution on the optimization of expected profit (see, e.g., [7]), but the problem of how to estimate demand parameters from low-volume sales data in the presence of stock-out substitution remains.

Much more work has been published in the field of dynamic pricing, where in one line of research pricing and inventory decisions are linked. See [3, 2, for surveys.

A common aspect of all cited papers (and papers cited there) that separates their research from ours is the following: those papers, in some sense, postulate the possibility to estimate a product's demand in an individual branch directly from sales data, in particular from sales rates. In our real-world application we have no replenishment, small delivery volumes per branch, lost sales with unknown or even no substitution, and sales rates depending much more on the success of the individual product at the time it was offered than on the size. Therefore, estimating future absolute demand data from historic sales data directly seems to need extra ideas, except maybe for the data aggregated over many branches.

Size optimization can be found in a few offers of retail optimization systems, like 7 thOnline (http://www.7thonline.com). It is not clear, on what kind of research these products are based and under which assumptions they work well. Our partner firm has checked several offers in the past and did not find any optimization tools that met their needs.

1.2. Our contribution. The main result of this work is: a useful forecast for the demand for sizes on the level of individual branches is too much to be asked for, but historic information about which sizes have been the scarcest and which sizes have been the amplest ones can be obtained by measuring the new Top-Dog-Index (TDI). The TDI can be utilized in a dynamic heuristic optimization procedure, that adjusts the size distributions in the branches' corresponding prepacks accordingly until the difference between the scarcest and the amplest sizes can not be improved anymore. The main benefit of the TDI: it measures the consistency of historic supply with sizes with the historic demand for sizes in a way that is not influenced by the attractivity 
of the product itself. This way, we can aggregate data over all products of a product group, thereby curing the problem with small delivery volumes per branch and product and size.

The potential of our TDI-approach is shown in a blind-study with 20 branches and one product group (womens' outer garments). Ten branches randomly chosen from the 20 branches (test-branches) received size pre-packs according to our heuristics' recommendations, ten received unchanged supply (control-branches). The result: One percentage point increase of gross yield per merchandise value for the test-branches against the control-branches. A conservative extrapolation of this result for our partner would already mean a significant increase of gross yield.

We have not seen any field study of this type documented in the literature so far. The only documented yield management studies in the apparel retail business (e.g., for dynamic pricing policies) try to prove the success of their methods by showing that they would have gained something on the set of data that was used to estimate the parameters of the model [1. Such tests are very far from reality: a by-product of our study is that in our case it makes absolutely no sense to compare gross yield data of a fashion discounter across seasons, because the differences between the yields in different seasons are much larger than the differences caused by anything we are interested in. This was the reason for us to use the parallel blind testing instead.

Further tests are planned by our partner on a larger scale in the near future.

1.3. Outline of the paper. In Section 2, we briefly restate the real-world problem we are concerned with. In Section 3 , we show our experience with straight-forward estimators for the demand distribution on sizes. In Section 4 , we introduce the new Top Dog Index, which is utilized in Section 5 in a heuristic optimization procedure. Section 6 is the documentation of a field study containing a blind testing procedure among two groups of branches: one supplied with and one supplied without the suggestions from the first step of the optimization heuristics. We summarize the findings in Section 7, including some ideas for further research.

\section{THE REAL-WORLD PROBLEM}

In this section, we state the problem we are concerned with. Before that we briefly provide the context in which our problem is embedded.

2.1. The supply chain of a fashion discounter. As in most other industries the overall philosophy of supply chain management in fashion retailing is to coordinate the material flow according to the market demand. The customer has to become the "conductor" of the "orchestra" of supply chain members. Forecasting the future demand is, therefore, crucial for all logistics activities. Special problems occur in cases like ours, when the majority of inventory items is not replenished, because the relationship between lead times and fashion cycles makes replenishments simply impossible. The resulting "textile pipeline" has strong interdependencies between marketing, procurement, and logistics.

The business model of our real world problem bases on a strict cost leadership strategy with sourcing in low cost countries, either East Asia or South East Europe. The transportation time is between one and six weeks, economies of scale are achieved via large orders.

2.2. Internal stock turnover of pre-packs. The material flow in our problem is determined by a central procurement for around 1200 branches. All items are delivered from the suppliers to a central distribution center, where a so called "slow cross docking" is used to distribute the items to the branches. Some key figures may give an impression of the situation: 32000 square meters, 80 workers, 30000 tons of garment in 10 million lots per year. Each branch is delivered once a week with the help of a fixed routing system. This leads to a sound compromise between inventory costs and costs of stock turnover. 
There are two extreme alternatives for the process of picking the items. The retailer can either work with one basic lot and deliver this lot or integer multiples of it for every article to the branches, or he develops individual lots for the shops. At the beginning of our analysis most of the articles were picked in one basic lot, but more than 40 other constellations were used additionally. The costs for picking the items are not relevant for the following analysis because only minor changes of internal processes are necessary. Only in later phases of our project a detailed analysis of the picking costs will be needed.

2.3. The problem under consideration. Recall that the stock turn-over is accelerated by ordering pre-packs of every product, i.e., a package containing a specified number of items of each size. We call the corresponding vector with a non-negative integral entry for every size a lot-type.

In this environment, we focus on the following problem: Given historic delivery data (in terms of pre-packed lots of some lot-type for each branch) and sales data for a group of products for each branch, determine for each branch a new lot-type with the same number of items that meets the relative demand for sizes more accurately.

In particular, find out from historic sales data some information about the relative demands for sizes. We stress the fact that stockout substitution in the data can not be neglected since replenishment does not take place (unsatisfied demand is lost and does not produce any sales data). We also stress the fact that we are not trying to improve the number of items delivered to each branch but the distribution of sizes for each branch only.

\section{Some Real-DAta AnAlysis EVAluating SEEMingly obVious APPROACHeS}

To deal with the problem described in Section 2 our partner has provided us some historic sales data for approximately 1200 branches over a time period of 12 months. The task was to forecast the size distribution of the future demand for each branch. A possible size distribution is depicted in Figure 1.

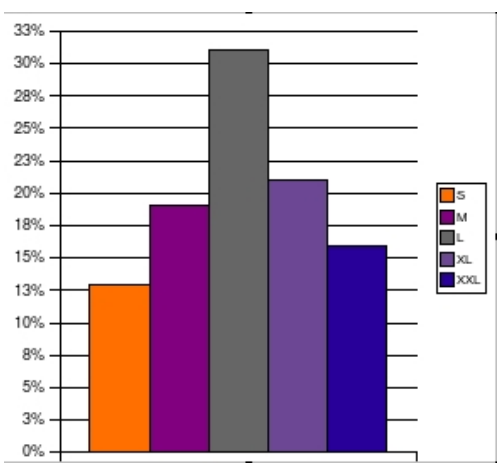

TABLE 1. A possible size distribution of the demand

The most obvious way to determine a size distribution as in Figures 1 is to count the number of sold items per size and divide by the total number of sold items. Here we have some freedom to choose the day of the sale where we measure the amounts. We have to balance two competing facets. An early measurement may provide sales figures which are statistically too small for a good estimate while a late measurement may suffer from unsatisfied demand that is not present in the sales data. 
The business strategy of our partner implies to cut prices until all items are sold. So, a very late measurement would only estimate the supply instead of the demand. As there is no expert knowledge to decide which is the optimal day of sales to count the amounts and estimate the size distribution we have applied a statistical test to measure the significance of the size distributions obtained for each possible day of counting the sold items.

Given a data set $D$, a day of sales $d$, and a size $s$ let $\phi_{s, d}(D)$ be the estimated demand for size $s$ measured on day $d$ as described above. We normalize so that $\sum_{s} \phi_{s, d}(D)=1$ for each day and each data set. Our statistical test partitions the original data set $D$ randomly into two disjoint data sets $D_{1}$ and $D_{2}$. Naturally we would not trust a forecast $\phi$ whenever $\phi_{s, d}\left(D_{1}\right)$ and $\phi_{s, d}\left(D_{2}\right)$ are too far apart. Statistically speaking $\phi$ would not be robust in that case. To measure more precisely how far apart $\phi_{s, d}\left(D_{1}\right)$ and $\phi_{s, d}\left(D_{2}\right)$ are, we define the discrepancy $\delta_{d}$ as $\delta_{d}\left(D_{1}, D_{2}\right):=\sum_{s}\left|\phi_{s, d}\left(D_{1}\right)-\phi_{s, d}\left(D_{2}\right)\right|$. In Figure 2 we have depicted the average discrepancy over all branches for the first 60 days of sale.

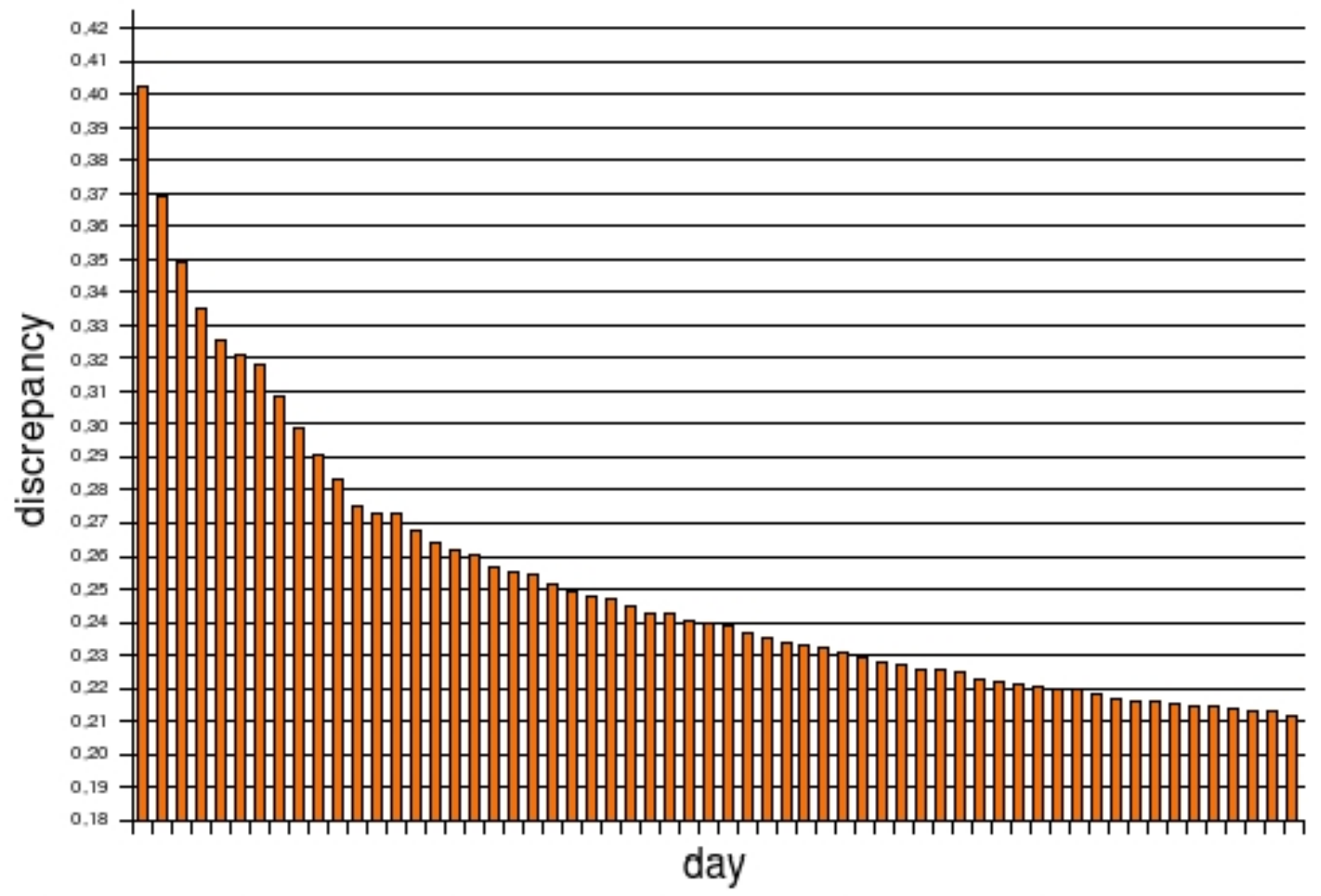

TABLE 2. Average discrepancy $\delta_{d}$ over all branches

For our practical application the significance of $\phi$ is too low for all possible days of measurement. We remark that in our examined data set the discrepancy $\delta_{d}$ tends to 0.19 as $d$ tends to the end of the sales period. An explanation why this obvious approach does not work in our case is due to the small sales numbers and the interference of product attractivity and price cutting strategies. 
Even when we tried to use only use ordinal information generated from an estimated size distribution (some size is too scarce, some size too ample), we encountered different results from different samples (same size was scarce in one sample and ample in the other).

In the same manner we have checked some common parametric models (mostly based on an estimation of a constant sales rate for each individual product-size pair) for demand forecasting on historic sales data from literature. None of them gives significant information of the size distribution of the demand of our data set, since the sales rates vary drastically and depend more on the attractivity of the products than on the sizes. For the details we refer to two diploma theses [5, 9] from our group.

\section{The Top-Dog-Index (TDI)}

In the previous section we learned that in our application, first, we cannot trust the common parametric models for the demand distribution and, second, measuring a size profile directly from sales data may lead to more or less random decisions. The main reason for the latter is the former and the interference between attractiveness of offered products and compatibility of offered sizes. Because of this, the stockout saturation of sales data happens at almost all times during the sales period, and thus aggregating the sales data of different products yields no reasonable information.

Our new idea throws overboard the desire to estimate an absolute size profile of the demand in every branch. Instead, we try to define a measure for the scarcity of sizes during the sales process that can be estimated from historic sales data in a stable way.

The following thought experiment is the motivation for our distribution free measure: Consider a product, for which in a branch all sizes are sold out at the very same day. This can be regarded as the result of an ideal balance between sizes in the supply. Our measure tries to quantify the deviation of this ideal situation in historic sales data. How can this be done? In the following, we extract data of a new type from the sales process.

Fix a delivery period $\Delta:=[0, T]$ from some day in the past normed to day 0 to day $T$. Let $B$ be the set of all branches that are operating in time interval $\Delta$, and let $P$ be the set of all products in a group delivered in time interval $\Delta$ in sizes from a size set $S$. We assume that in each branch the product group can be expected to have homogeneous demand for sizes throughout the time period. Fix $b \in B$. For each $p \in P$ and for each $s \in S$ let $\theta_{b}(p, s)$ be the stockout-day of size $s$ of product $p$, i.e., the day when the last item of $p$ in size $s$ was sold out in branch $b$.

Fix a size $s \in S$. Our idea is now to compare for how many products $p$ size $s$ has the earliest stockout-day $\theta(p, s)$ and for how many products $p$ size $s$ has latest stockout-day $\theta(p, s)$. These numbers have the following interpretation: If for many more products the stockout-day of the given size was first among all sizes, then the size was scarce. If for many more products the stockout-day of the given size was last among all sizes, then the size was ample.

In order to quantify this, we use the following approach. (In fact, it is not too important how we exactly quantify our idea, since we will never use the absolute quantities for decision making; we will only use the quantities relative to each other.)

Definition 4.1 (Top-Dog-Index). Let $s \in S$ be a size and $b$ be a branch. The Top-Dog-Count $\mathrm{W}_{b}(s)$ for $s$ in $b$ is defined as

$$
\mathrm{W}_{b}(s):=\left|\left\{p \in P \mid \theta_{b}(p, s)=\min _{s^{\prime} \in S} \theta_{b}\left(p, s^{\prime}\right)\right\}\right|
$$

and the Flop-Dog-Count $\mathrm{L}_{b}(s)$ in $b$ is defined as

$$
\mathrm{L}_{b}(s):=\left|\left\{p \in P \mid \theta_{b}(p, s)=\max _{s^{\prime} \in S} \theta_{b}\left(p, s^{\prime}\right)\right\}\right| .
$$


Moreover, for a fixed dampening parameter $C>0$ let

$$
\operatorname{TDI}_{b}(s):=\frac{\mathrm{W}_{b}(s)+C}{\mathrm{~L}_{b}(s)+C}
$$

be the Top-Dog-Index (TDI) of Size $s$ in Branch $b$.

In the data of this work, we used $C=15$.

4.1. Statistical significance of the Top-Dog-Index. In a similar way as in Section 3 we want to analyze the significance of the proposed Top-Dog-Index. Since this method is supposed to be applied to a real business case, we analyze the statistical significance in more detail. Instead of two data sets $D_{1}$ and $D_{2}$ as in Section 3 we utilize seven such sets $D_{i}$. Therefore, we assign a random number in $\{1,2,3,4\}$ to each different product. The sets are composed of the data of products where the corresponding random number lies in a characteristic subset of $\{1,2,3,4\}$. See Table 3 for the assignment. For the interpretation we remark that the pairs $\left(D_{1}, D_{2}\right),\left(D_{3}, D_{4}\right)$, and $\left(D_{5}, D_{6}\right)$ are complementary. The whole data set is denoted by $D_{7}$.

\begin{tabular}{cc}
\hline$D_{1}$ & $\{1,2\}$ \\
$D_{2}$ & $\{3,4\}$ \\
$D_{3}$ & $\{1,3\}$ \\
$D_{4}$ & $\{2,4\}$ \\
$D_{5}$ & $\{3\}$ \\
$D_{6}$ & $\{1,2,4\}$ \\
$D_{7}$ & $\{1,2,3,4\}$ \\
\hline
\end{tabular}

TABLE 3. Assignment of test sets

Since the Top-Dog-Index is designed to provide mainly ordinal information, we have to use another statistical test to make sure that it yields some significant information. Let $\operatorname{TDI}_{b}\left(s, D_{i}\right)$ denote the Top-Dog-Index in Branch $b$ of Size $s$ computed from the data in Data Set $D_{i}$. We find it convincing to regard the ordinal information generated by the Top-Dog-Index as robust whenever we have

$$
\mathrm{TDI}_{b}\left(s, D_{i}\right) \gg \operatorname{TDI}_{b}\left(s^{\prime}, D_{i}\right) \Longleftrightarrow \operatorname{TDI}_{b}\left(s, D_{j}\right) \gg \mathrm{TDI}_{b}\left(s^{\prime}, D_{j}\right)
$$

for each pair of sizes $s, s^{\prime}$ and each pair of data sets $D_{i}, D_{j}$. In words: the order of Top-DogIndices of various sizes does not change significantly when computed from a different sample. The following is a sufficient condition for this to happen:

$$
\frac{\mathrm{TDI}_{b}\left(s, D_{i}\right)}{\sum_{j} \mathrm{TDI}_{b}\left(s, D_{j}\right)} \approx \text { const }_{i}
$$

Our first aim is to provide evidence that the $\operatorname{TDI}_{b}(s)$ values are robust measurements in this sense. There is a nice way to look at equation 5 graphically. Let us fix a size $s$. For each branch $b$ let us plot a column of the relative values $\frac{\operatorname{TDI}_{b}\left(s, D_{i}\right)}{\sum_{j} \operatorname{TDI}_{b}\left(s, D_{j}\right)}$ for all branch-size combinations and for all $i$. The columns corresponding to the same branch-size combination but different samples are stacked on top of each other. This way, each colum stack has height one in total, and the size relations of the measurements based on the different samples can be assessed right away. To show the robustness of the mean and the median, we have added an additional column for each of them: The median of the seven estimates corresponds to the height of the top-most column, the mean to the height of the second-to-top-most column. The goal now is not to read off the exact values, but to get an intuitive impression how heavy an estimate depends on the data subset it was computed from. 
The Top-Dog-Indices are plotted this way in Figure 1.

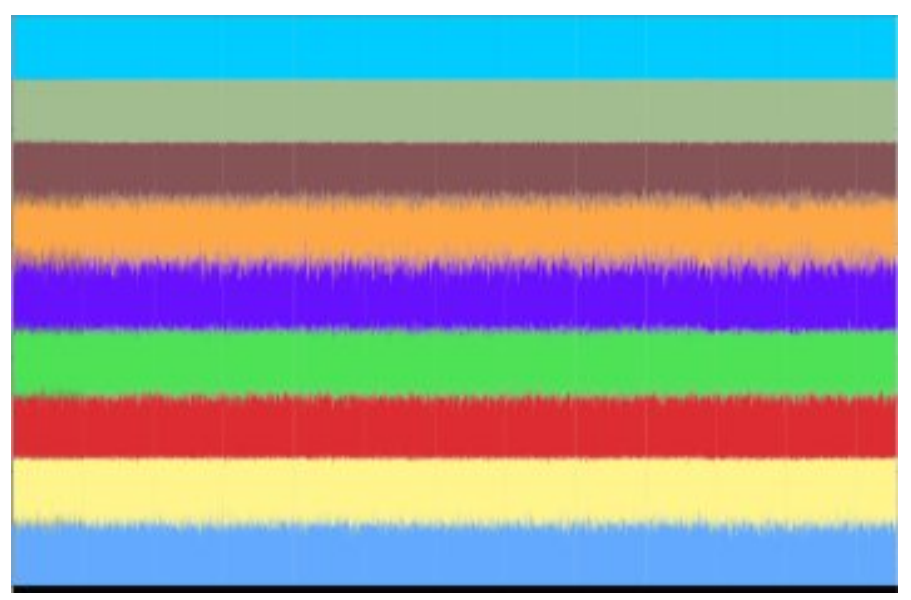

Figure 1. Relative distribution of the Top-Dog-Index on seven data subsets for all branch-size combinations; the two top-most columns are median and mean, resp.
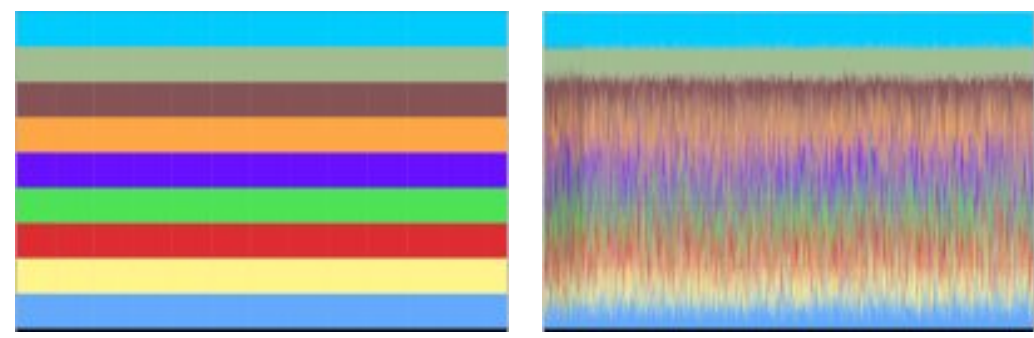

FiguRE 2. Relative distribution of deterministic and random numbers on seven data subsets and branches; the two top-most columns are median and mean, resp.

In order to provide some intuitively clear reference data to compare to the plot of Figure 1, we present the corresponding plots for the two extreme cases of deterministic numbers (i.e., $\operatorname{TDI}_{b}\left(s, D_{i}\right)=\operatorname{TDI}_{b}\left(s, D_{j}\right)$ for all $i$ and $\left.j\right)$ and totally random numbers in Figure 2 In the complete deterministic case the areas of same color form perfect rectangles. In the random case the areas of same color corresponding to the data subsets form zig-zag lines; the median has fewer zig-zag than the mean, but both are quite stable because the random numbers are all from the same distribution.

It is immediately obvious that the plot of Figure 1 looks more like the plot in the deterministic case as the plot in the random case. It is interesting to note that the dampening parameter in the computation of the TDI does indeed influence the amount of noise in the plot but had almost no influence on the order of TDI values, which is what we indend to use.

Recall that we have analyzed the direct estimation of size distributions for each branch by looking at two complementary samples of the data set. In order to be able to directly compare the results of that attempt to Figure 1, we plot the size distributions for the same seven data 


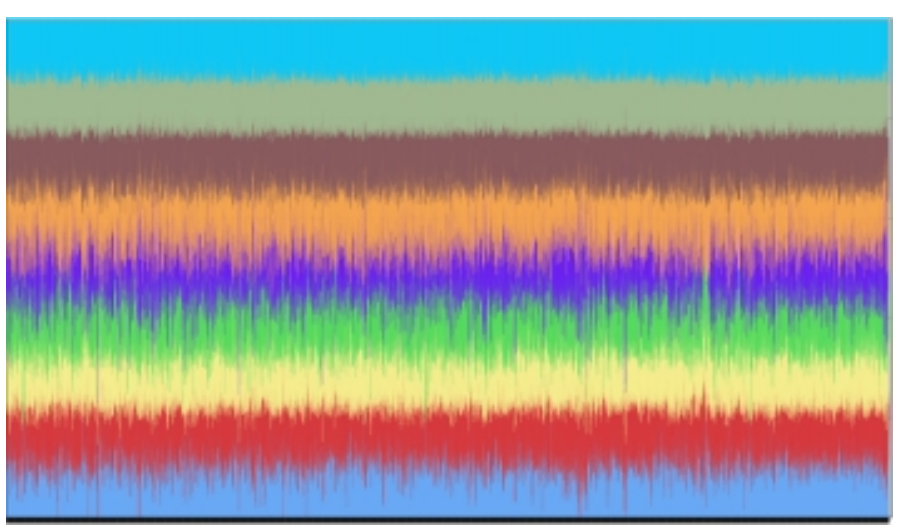

FiguRE 3. Relative distribution of sales up to Day 0 on on seven data subsets for all branch-size combinations; the two top-most columns are median and mean, resp.

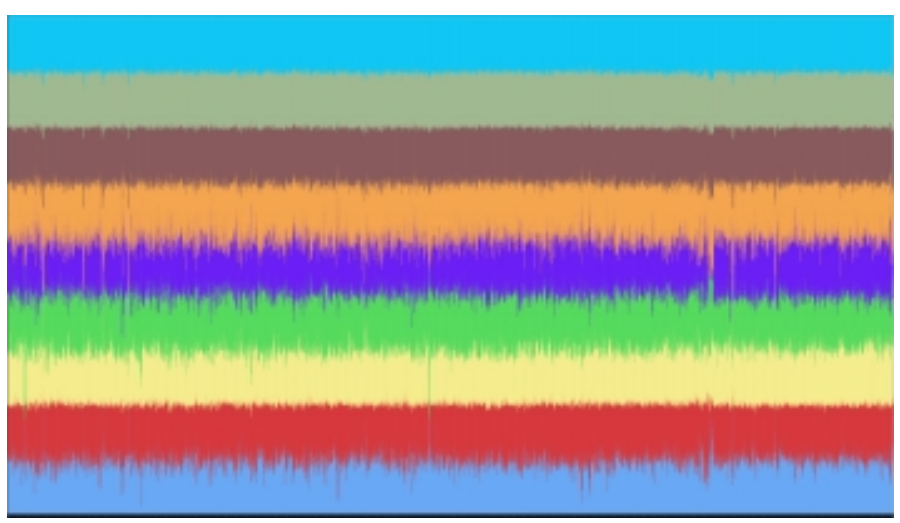

FiguRE 4. Relative distribution of sales up to Day 12 on on seven data subsets for all branch-size combinations; the two top-most columns are median and mean, resp.

subsets. This is shown in Figure 3 for an estimate from the sales up to Day 0 (the first day in the sales period) and in Figure 4 for an estimate from the sales up to Day 12.

It can be seen that Day 0-estimates are extremely dependent on the data subset even if only the relative values are taken into account, i.e., the estimates are not robust even in the weak sense measured in the plot. The Day-12 estimates are more robust, but not even close to what the TDI achieves in Figure 1. Moreover, as we said, those estimates are already quite close to the supply because of unsatisfied demand, and will therefore fail to measure the size distribution of the demand.

Generating a robust statistics for a set of data is, of course, easy: just assign the same deterministic number to each object. This is not what we want since this does not carry any useful information. We claim that the Top-Dog-Index exhibits differences between demands for sizes in each branch individually. That this is indeed the case, follows from the Top-Dog-Indices that we encountered in the field study documented below. But let us first discuss which actions we could take to improve the size distributions of the branches' supply. 
So far we have argued that the Top-Dog-Index produces size-related information in a robust way, while other methods fail (at least for our given real-world data). In the next section we describe a procedure to harmonize demand and supply with respect to the size distribution. In Section 6 we provide evidence via a real-world blind study that this procedure helps to raise the gross yield in reality; thus, the Top-Dog-Index is correlated to the size distribution of the demand.

\section{The HeURistic SizE OPTIMIZATION PROCEDURE BASED ON THE TDI}

Interesting for us is not the absolute TDI of a size but the TDIs of all sizes in a branch compared to each other, i.e., the ordinal information implied by the TDIs. The size with the maximal TDI among all sizes can be interpreted as the scarcest (the one that was sold the fastest) size in that branch; the size with the minimal TDI among all sizes can be interpreted as the amplest size in that branch. Of course, we have the problem of deciding whether or not a maximal TDI is significantly larger than the others. Since the absolute values of the TDIs have no real meaning we did not even try to assess this issue in a statistically profound way.

Our point of view is again that absolute forecasting is too much to be asked for. Therefore, we resort to a dynamic heuristic optimization procedure: sizes with "significantly" large TDIs (Top-Dog-Sizes) should receive larger volumes in future deliveries until their TDIs do not improve anymore, while sizes with "significantly" small TDIs (Flop-Dog-Sizes) need smaller supplies in the future. Whenever this leads to oversteering, the next TDI analysis will show this, and we go back one step. This is based on the assumption that the demand for sizes does not change too quickly over time. If it does then optimization methods based on historic sales data are useless anyway.

Let us describe our size distribution optimization approach in more detail.

We divide time into delivery periods (e.g., one quarter of a year). We assume that the sales period of any product in a delivery period ends at the end of the next delivery period (e.g., half a year after the beginning of the delivery period). Recall that a size distribution in the supply of a branch is given by a pre-pack configuration: a package that contains for each size a certain number of pieces of a product (compare Section 2.2).

We want to base our delivery decisions for an up-coming period on

- the pre-pack configuration of the previous period and

- the TDI information of the previous period giving us the deviation from the ideal balance

According to given restrictions from the distribution system, we assume that only one pre-pack configuration per branch is allowed. We may use distinct pre-pack configurations for different branches, though.

Since we are only dealing with the size distribution of the total supply but not with the total supply for a branch itself, the total number of pieces in a pre-pack has to stay constant. Since the TDI information only yields aggregated information over all products in the product group, all products of this group will receive identical pre-pack configurations in the next period, as desired.

In order to adjust the supply to the demand without changing the total number of pieces in a pre-pack, we will remove one piece of a Flop-Dog-Size from the pre-pack and add one piece of a Top-Dog-Size instead. At the end of the sales period (i.e., at the end of the next delivery period), we can do the TDI-analysis again and adjust accordingly.

Given the usual lead times of three months this leads to a heuristics that reacts to changes in the demand for sizes with a time lag of nine month to one year. Not exactly prompt, but we assume the demand for sizes to be more or less constant over longer periods of time. 
The most interesting question for us was how much, in practice, could be gained by performing only one step of the heuristics explained above.

\section{A REAL-WORLD BLIND STUDY}

In this section we describe the set-up and the results of the blind study carried out by our partner. A summary of parameters can be found in Table 4 .

\begin{tabular}{|c|c|}
\hline Test period : & April through June 2006 (3 Months) \\
\hline Data collection period: & April through September 2006 (6 Months) \\
\hline Branches: & $\begin{array}{l}20 \text { branches with unbalanced TDIs } \\
\text { randomly classified into } 10 \text { test and } 10 \text { control branches }\end{array}$ \\
\hline $\begin{array}{l}\text { Pieces of merchandise: } \\
\text { Merchandise value: }\end{array}$ & $\begin{array}{l}\text { approx. } 4000 \text { pieces for all test and control branches } \\
\text { approx. } 30000 €\end{array}$ \\
\hline
\end{tabular}

TABLE 4. Summary of parameters of the blind study

6.1. Selection of branches. A reasonable selection of branches for a test and a control group had to meet essentially three requiremens: first, only those branches should be chosen whose TDI indicated that, in the past, the supply by sizes did not meet the demand for sizes; secondly, no branch should be chosen, where other tests were running during the test period; thirdly, the assignment of branches to test- and control group should be completely random. The reason for the third aspect was that this way all other influences on the gross yield than the selection of sizes would appear similarly in both the test group and the control group and, thus, would average out evenly.

We suggested a set of 50 branches with interesting Top-Dog-Indices to our partner. Out of these 50 branches, our partner chose 20 branches where a potential re-packing of pre-packs would be possible. This set of 20 branches was fixed as the set of branches included in our blind study.

After that, a random number between 0 and 1 was assigned to each of the 20 branches. The 10 branches with the smallest random numbers were chosen to be the test group, the rest was taken as the control group.

6.2. Handling of pre-packs. Next, we had to specify the modifications to the size distribution in pre-packs on the basis of the TDI information. It turned out that additional side constraints had to be satisfied: Whenever a product would appear in an advertisement flyer, the pre-pack had contained at least one piece in each of the four main sizes S, M, L, and XL. That means, although sometimes the TDI suggested that $\mathrm{S}$ was the amplest size, we could not remove the only piece in S from the pre-pack. We removed a piece of the second amplest size (M or L, of which there were two in the unmodified pre-packs) instead. To all branch deliveries, one additional piece of XL was packed, because this was the scarcest size in every branch in the test group. This way, the total number of pieces was unchanged in every pre-pack, as suggested in Section 5 .

Since all orders had been placed well before the decision to conduct a blind study, our partner re-packed all pre-packs for the test branches according to Table 5 .

6.3. Time frame. The test included two relevant time periods: the first period from which the TDI data was extracted and the test period in which the recommendations based on the TDI data were implemented for the test group.

The TDI data was drawn from a delivery period of nine month (January through September 2005) and a sales period of twelve month (January through December 2005).

The test data was drawn from a delivery period of three months (April through June 2006) and a sales period of six months (April through September 2006). 


\begin{tabular}{|c|c|c|c|c|}
\hline \multirow{2}{*}{$\begin{array}{l}\text { test group } \\
\text { Branch }\end{array}$} & \multicolumn{2}{|c|}{$\begin{array}{c}\text { special } \\
\text { advertising }\end{array}$} & \multicolumn{2}{|c|}{$\begin{array}{c}\text { no } \\
\text { advertising }\end{array}$} \\
\hline & remove & add & remove & add \\
\hline 1 & $\mathrm{~L}$ & XL & $\mathrm{L}$ & XL \\
\hline 2 & M & XL & M & $\mathrm{XL}$ \\
\hline 3 & $\mathrm{~L}$ & XL & $\mathrm{S}$ & XL \\
\hline 4 & $\mathrm{~L}$ & XL & $\mathrm{S}$ & XL \\
\hline 5 & M & XL & M & $\mathrm{XL}$ \\
\hline 6 & M & XL & M & $\mathrm{XL}$ \\
\hline 7 & M & XL & M & XL \\
\hline 8 & $\mathrm{~L}$ & $\mathrm{XL}$ & $\mathrm{S}$ & $\mathrm{XL}$ \\
\hline 9 & M & XL & M & XL \\
\hline 10 & $\mathrm{M}$ & XL & M & XL \\
\hline
\end{tabular}

TABLE 5. How the pre-packs were modified in the test group

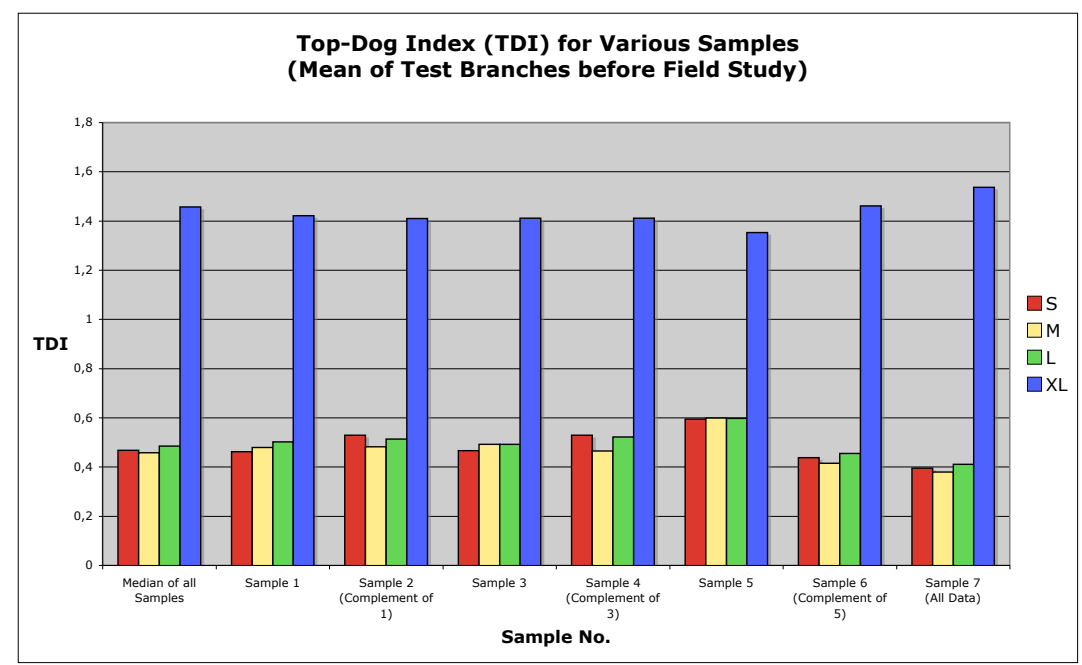

FiguRE 5. TDI in the test branches from historic sales data

6.4. Data collection. In order to sort out contaminated data easily, our partner agreed to take stock to check inventory data for correctness every month. To receive a good estimate for the financial benefit of the supply modification proposed by the procedure described in the previous section we had defined some criteria how to detect contaminated data automatically via a computer program.

6.5. Data analysis. The initial situation was given by the data set described in Section 3 . In Figure 5 we have depicted the initial Top-Dog-Indices for the test branches and in Figure 6 the 


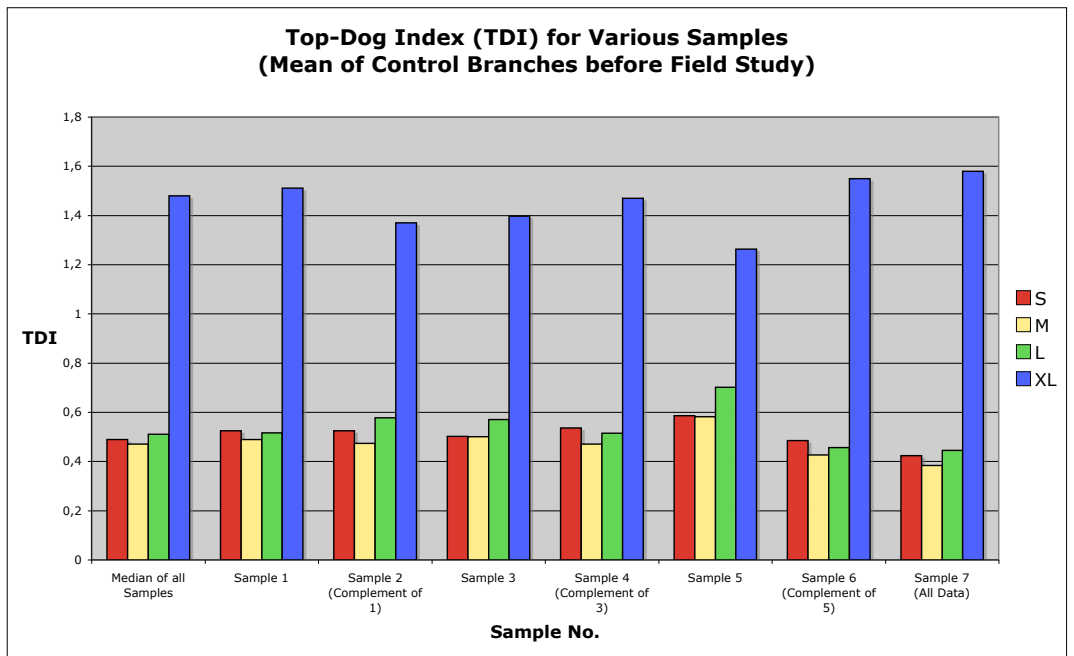

FiguRE 6. TDI in the control branches from historic sales data

initial Top-Dog-Indices for the control branches. Moreover, Figures 7 and 8 finally show the situations for the individual branches.

The analysis of our field study was intended to answer the following two main questions: are the Top-Dog-Indices better distributed in the test branches than in the control branches, and, if yes, does this have a significant monetary impact?

To investigate the latter, we had to analyze some monetary variables. The most important monetary indices for our partner are the gross yield and the last price. The gross yield directly shows how much turnover was lost using a price cutting strategy to sell out all items. The last price tells us how far one was forced to dump items provoked by an inadequate size distribution of the supply. Since the values of different products vary widely we only consider relative values. So, the gross yield is given by the ratio of realized turnover and theoretic revenue without cutting the prices.

Since we had to deal with a large amount of lost or inconsistent data, we have applied two ways of evaluating gross yield and last price. Imagine that your data says that you have sold 10 items of a given article in a given branch but that the supply was only 8 items. Or the other way round that the initial supply was 10 items, during the time 8 items were sold, but all items are gone. Both of the described situations occurred significantly often in our data set.

Our first strategy to evaluate the given data was to ignore inconsistent data. In the first case, 8 sale transactions are consistent. For the remaining two items the corresponding supply transaction is missing. So, we simply ignore these transactions. In the other case we would ignore the supply of two items.

The alternative to ignoring inconsistent data is to estimate or recover it from the rest of the data set. As an example, we would simply assume that there was a supply of 10 items instead of 8 items at the same price level in the first case. In the second case we would assume that the 


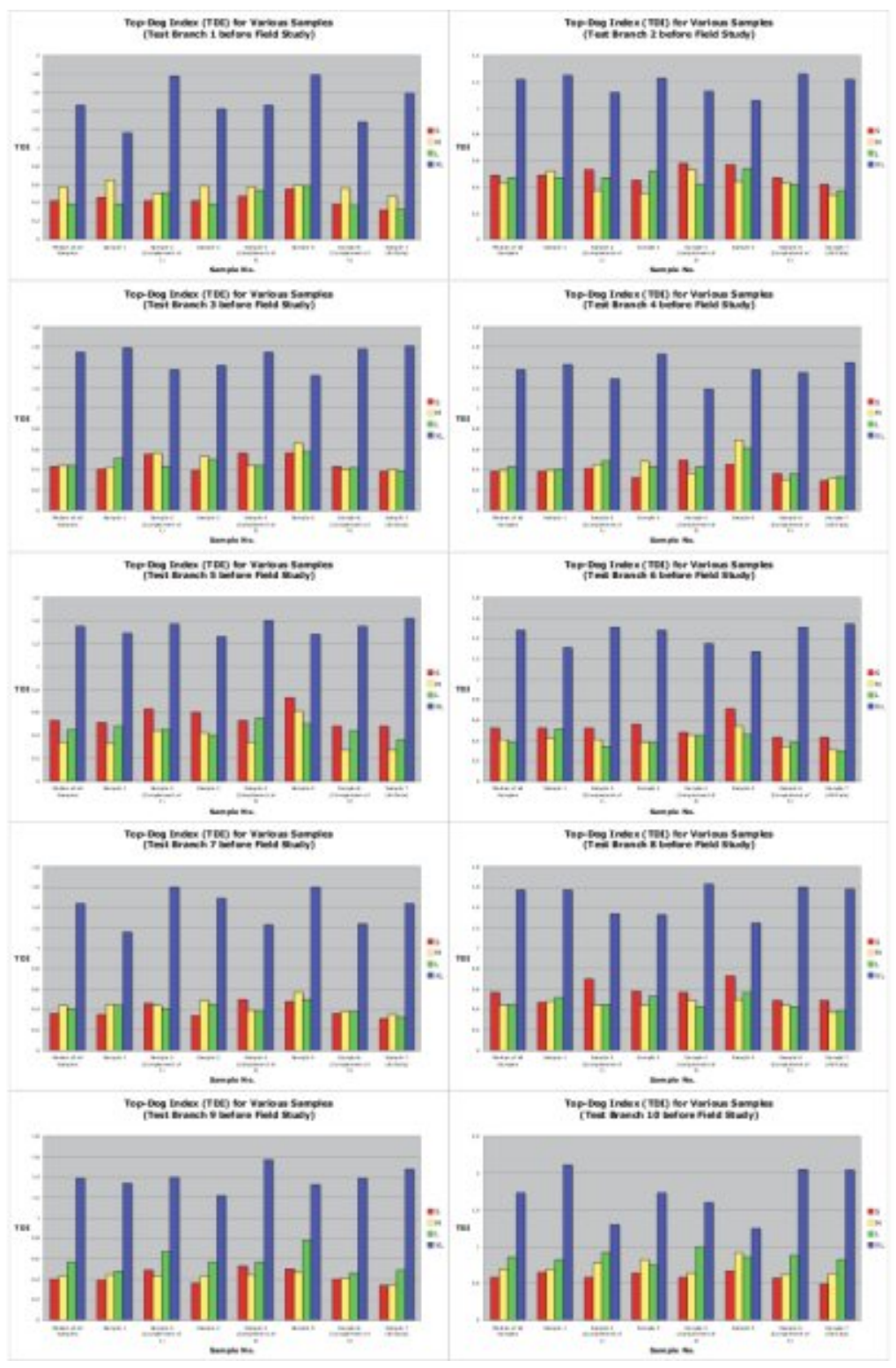

FiguRE 7. Individual TDIs in test branches from historic sales data

remaining 2 items were also sold. Maybe they were shoplifted, some sort of selling for a very cheap prize. So, we need an estimation for the selling prize of the two missing items. Here, we have used the last selling price over all sizes for this product in this branch as an estimate.

Neither evaluation method reflects reality exactly. Our hope was that both estimations encompass the true values. At least our partner accepts both values as a good approximation of reality. The truth may be somewhere in between both values. 


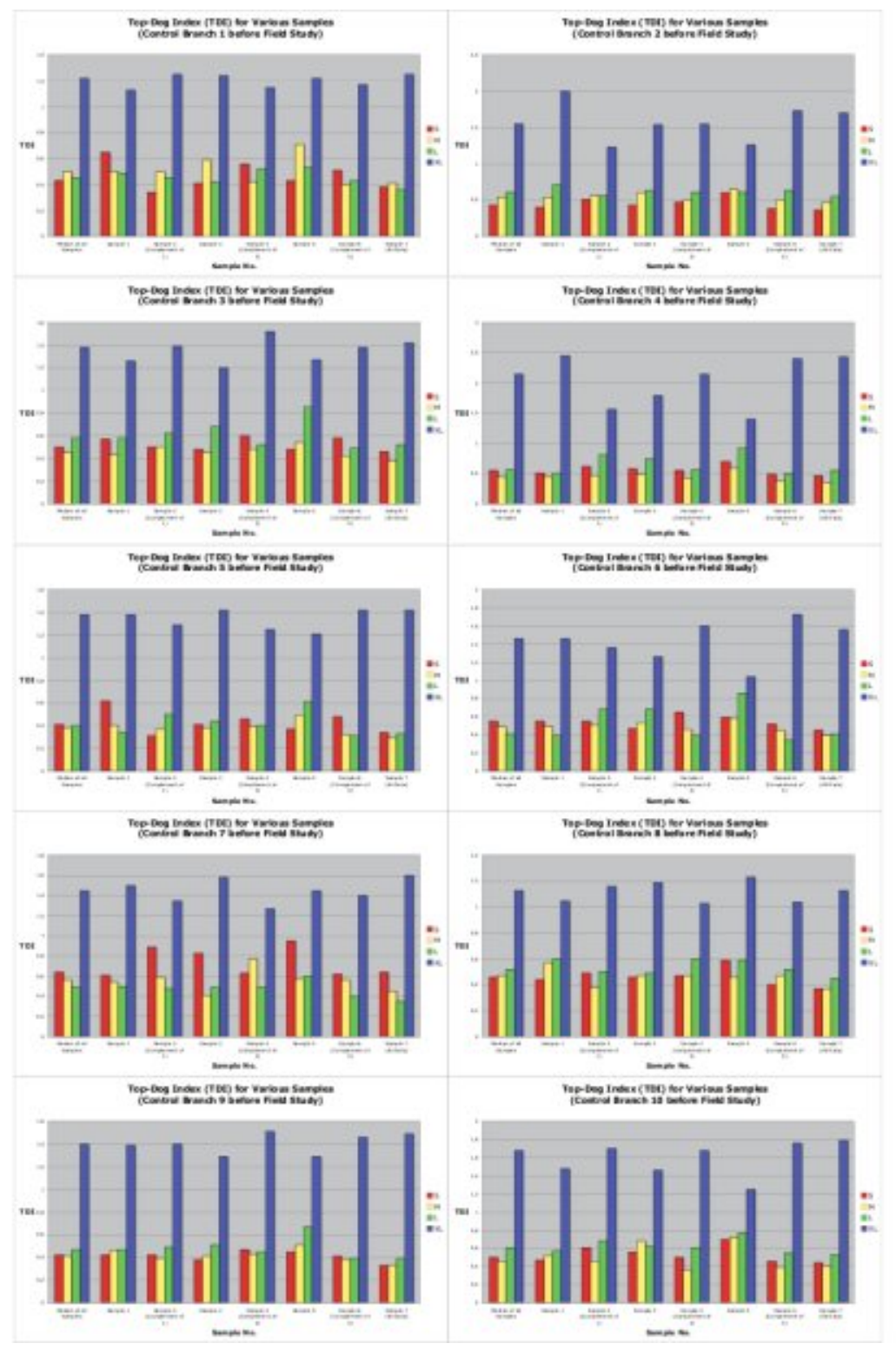

FIGURE 8. Individual TDIs in control branches from historic sales data

6.6. Results. We have depicted the new Top-Dog-Indices after applying our proposed repacking in Figure 9 for the test branches and in Figure 10 for the control branches. Figures 11 and 12 finally show the results for the individual branches.

We can see in Figure 8 and 12 that the absolute Top-Dog-Indices are not as constant over seasons as we would wish. However, the induced order on sizes seems quite stable accross seasons, and this is all we try to exploit.

We can see that it is rather hard to compare the Top-Dog-Indices of the same branches before and after the blind study. The situation on the real market almost never stays constant over time. 


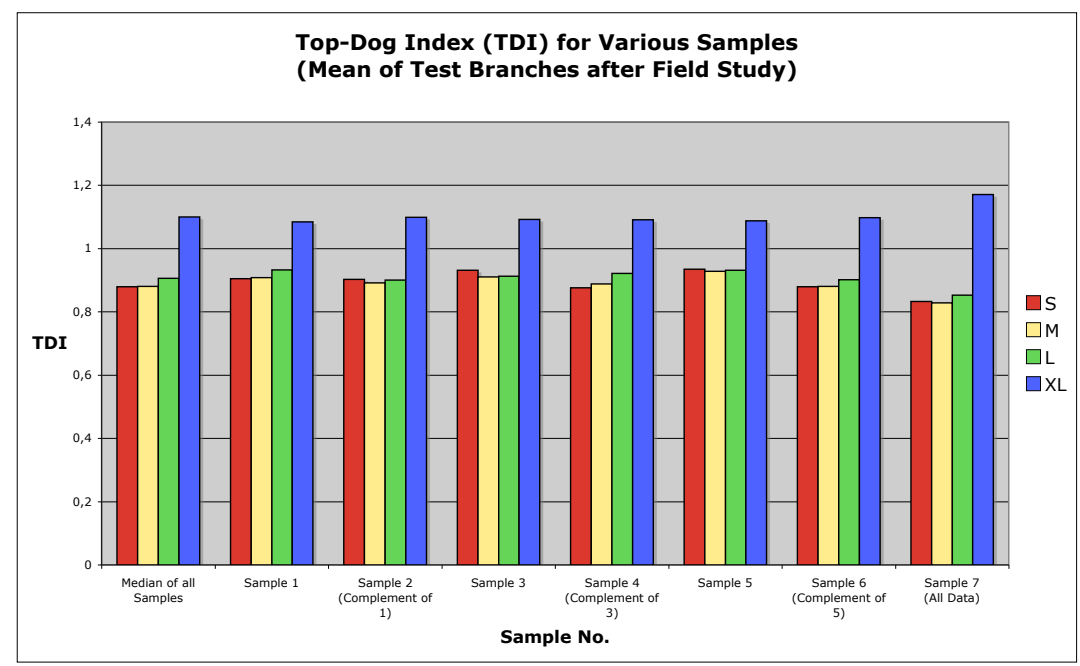

FIGURE 9. TDI in the test branches in test period

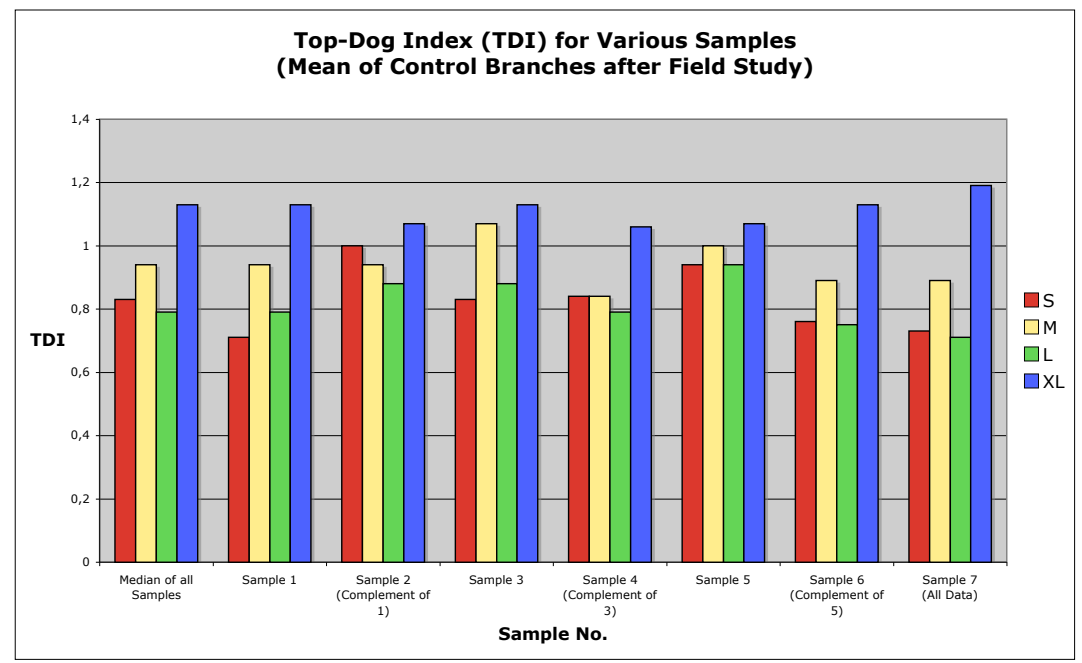

Figure 10. TDI in the control branches in test period 


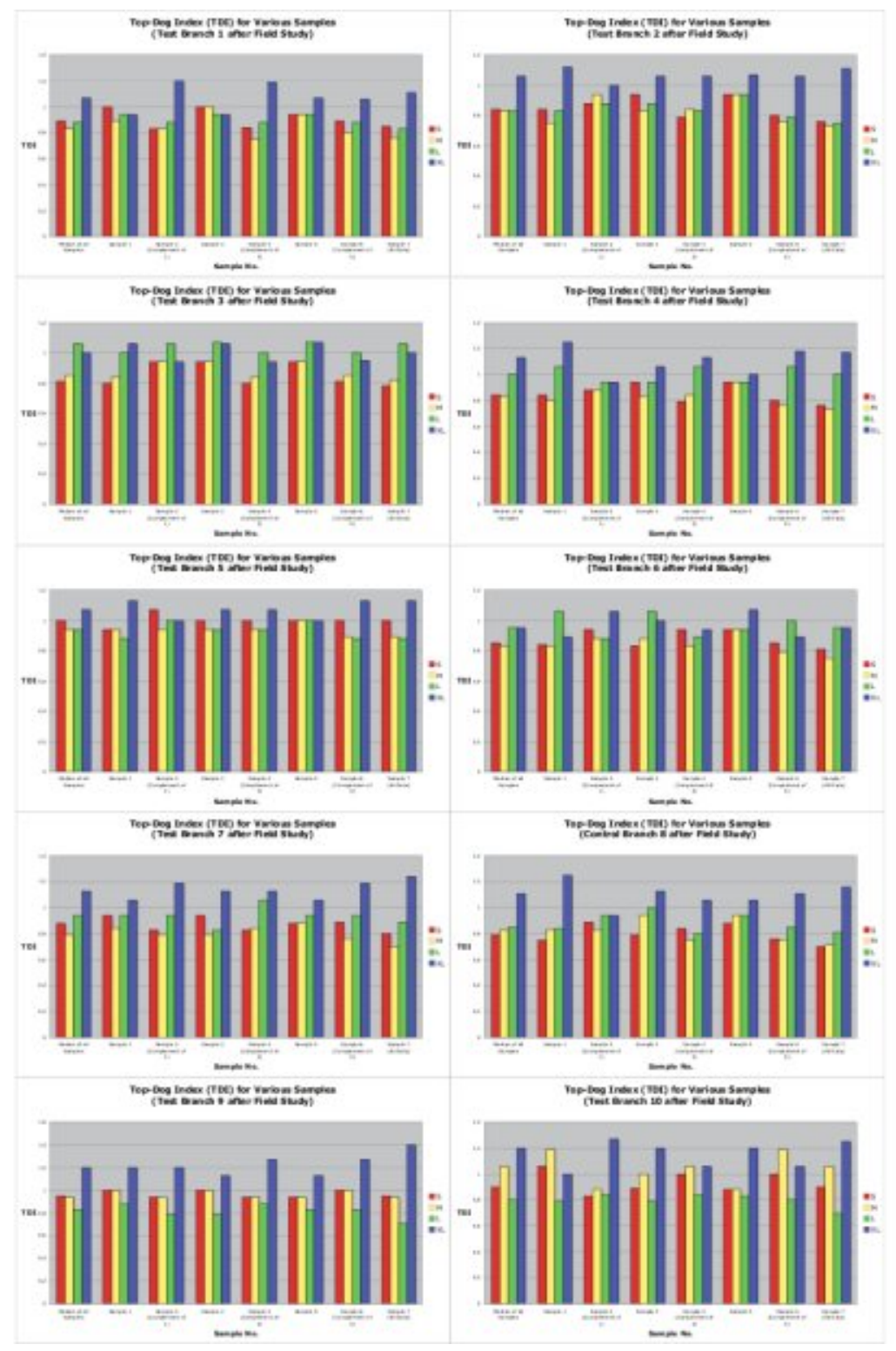

FIGURE 11. Individual TDIs in test branches in test period

There are so many influences not considered in our study that it would have been a bad idea to measure a possible raise of earnings directly. For this reason, the simultaneous observation of a test group and a control group makes all outer effects appear in both.

Comparing Figure 9 and Figure 10 based on the same time period, it appears that the TopDog-Indices of the test group have improved more.

More specifically, in Figures 11 and 12 we see that in some of the test branches (especially, Test Branches 5 and 6), Size XL is no longer too scarce, while it remains too scarce in other 


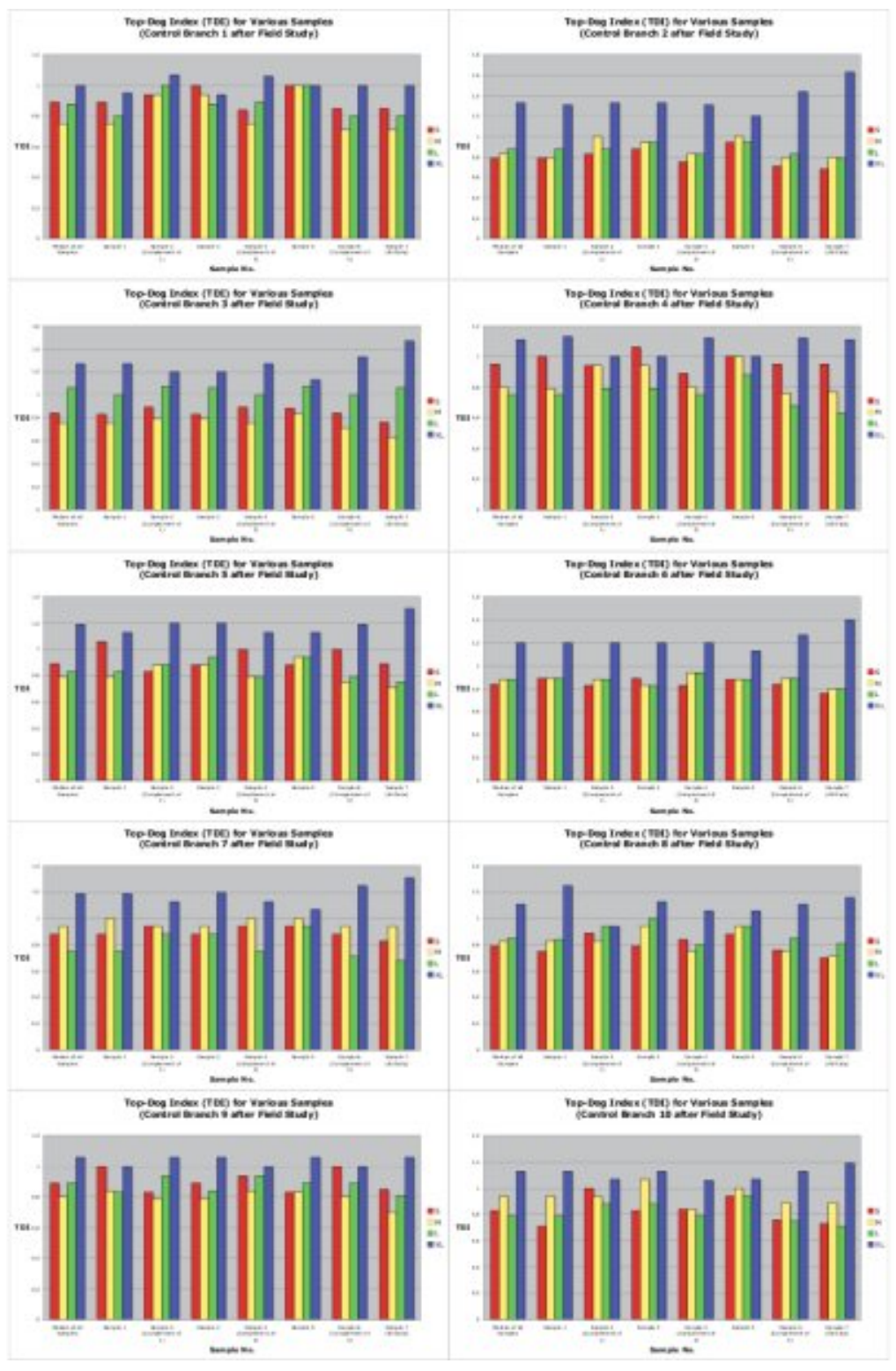

FIGURE 12. Individual TDIs in control branches in test period

branches. Moreover, on average over all test branches, the Top-Dog-Indices of Sizes S, M, and L are better balanced, while in the control branches the corresponding Top-Dog-Indices differ.

While the former achievement might have been equally possible on the basis of a statistics aggregated over all branches, it seems that the latter result was made possible only by the branch dependent information from the Top-Dog-Index, since different sizes were removed from the prepacks in favor of XL. Moreover, some of the test branches still need fewer pieces in Size XL, some test branches don't. That is, in the next optimization step, the branch dependent information becomes vital also for the supply with Size XL. 
But is an improved Top-Dog-Index really an improvement for the business? To answer this question, we have quantified the gross yields and the last prices in the test group and the control group, resp.

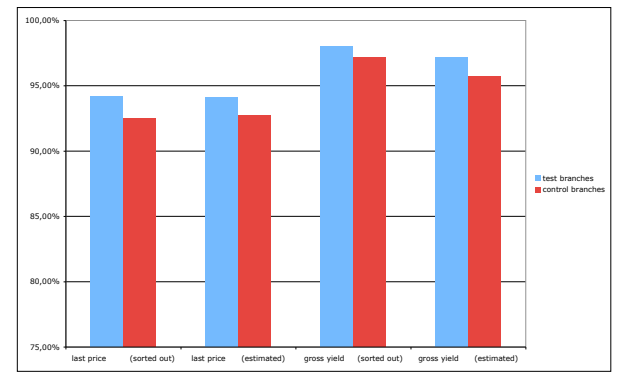

Figure 13. Average last prices/gross yields per merchandise value

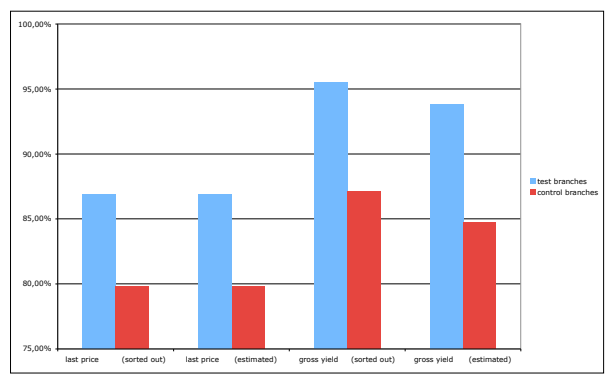

Figure 14. Minimal last prices/gross yields per merchandise value

In Figure 13 we have compared the average values of the gross yield and the last price for the control and the test branches for both evaluation methods. The gross yield of the test branches is $98.0 \%$ using the ignore and $97.2 \%$ using the estimate method. For the control branches we have gross yields of $97.2 \%$ (ignore) and $95.7 \%$ (estimate). This corresponds to improvements of 0.85 and 1.5 percentage points, resp.

The improvement for the last price is even larger. The test branches show a last price level of $94.2 \%$ (ignore) and $94.1 \%$; the control branches exhibit a last price level of $92.5 \%$ (ignore) and $92.7 \%$ (estimate). This corresponds to improvements of 1.7 and 1.4 percentage points, resp.

The drastically improved results for the respective "loser branches" (see Figure 14) and the reduced standard deviation (see Figure 15) in the data of the test branches provides evidence for the fact that our procedure was able to reduce the risk of a very low last price or a very low gross yield in an individual branch. This effect is desirable beyond the better earnings, as very low last prices undermine the image of the retailer. 


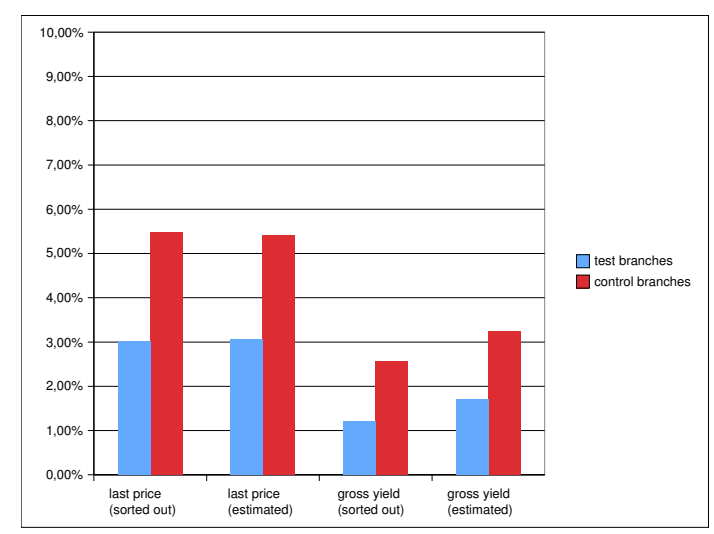

FIGURE 15. Standard deviation of last prices/gross yields per merchandise value

6.7. Statistical evidence of the improvement of the gross yield. As in the previous sections we analyze our results concerning the gross yield from the statistical point of view. Faced with the fact of a widely varying gross yield over the branches with no appropriate theoretic sales model, we have to restrict ourselves to distribution-free statistics.

Therefore, we adapt the Wilcoxon rank sum test to our situation. This is a test method to find out whether or not two data sets are drawn from the same distribution. We sort in increasing order the gross yields of the 20 branches participating in our blind study. We associate the largest value with Rank 1, the second largest with Rank 2, and so on. Then we form the rank sums of the test branches and the control branches, resp. The more the rank sums differ, the less likely is the event that our method did not influence the gross yields/last prices at all.

For the Wilcoxon rank sum test, it is vital that we have partitioned the 20 branches for the blind study independently at random into test and control branches.

It is intuitively clear that a smaller rank sum for the gross yield/last price is more likely if the corresponding expected values are better. A lower rank sum can indeed occur by pure coincidence, but the probability decreases with the rank sum. As an example, the rank sum for the test branches regarding the gross yield measured by the ignore method is 89 . If we had not changed anything, the chance to receive a rank sum of 89 or lower would have been $12.4 \%$. So, we have a certainty of $87.6 \%$ that our proposed re-packing improved the situation. (More formally: the probability that the gross yields of the test branches and the gross yields of the control branches stem from the same distribution, i.e., nothing has changed systematically, is at most $12.4 \%$.)

Now we consider different scenarios. Let $y_{i}(b)$ be the gross yield measured with the method ignore of branch $b$ and $y_{e}(b)$ the gross yield measured with the method estimate. By $i_{c}$ we denote the scenario where we consider the values $y_{i}(b)$ for control branches and the values $y_{i}(b)+\frac{c}{100}$ for test branches. Similarly, we define the scenarios for $e_{c}$ utilizing $y_{e}(b)$ instead of $y_{i}(b)$. In Table 6 we have given the rank sums and the certainties of some scenarios.

How can we interprete these numbers? The first two columns of Table 6 show that with a certainty of $87.6 \%$ (ignore) and $97.4 \%$ (estimate) that our proposed modification increased the expected gross yield. In Scenario $i_{-0.25}$ we artificially decrease the gross yield (ignore) values by 0.25 percentage points. The monetary value associated with this specific decrease can be interpreted, e.g., as the implementation and consultancy costs of the modification. So, by a look at Table 6 we can say that with a certainty of $82.4 \%$ our proposed modification yields an improvement of the gross yield (ignore) by at least 0.25 percentage points. 


\begin{tabular}{|r|rrrrrrrrrr|}
\hline & $i_{-0.00}$ & $e_{-0.00}$ & $i_{-0.25}$ & $e_{-0.25}$ & $i_{-0.50}$ & $e_{-0.50}$ & $i_{-0.75}$ & $e_{-0.75}$ & $e_{-1.00}$ & $e_{-1.50}$ \\
\hline control group & 121 & 131 & 118 & 128 & 105 & 124 & 94 & 120 & 112 & 107 \\
test group & 89 & 79 & 92 & 82 & 105 & 86 & 116 & 90 & 98 & 103 \\
certainty (\%) & 87.6 & 97.4 & 82.4 & 95.5 & 48.5 & 91.7 & 19.7 & 86.0 & 68.5 & 54.4 \\
\hline
\end{tabular}

TABLE 6. Ranks sums and certainties of improvements of the gross yield

\section{Conclusion and outlook}

The distribution of fashion goods to the branches of a fashion discounter must meet the demand for sizes as accurately as possible. However, in our business case, an estimation of the relative demand for apparel sizes from historic sales data was not possible in a straight-forward way.

Our proposal is to use the Top-Dog-Index (TDI), a measure that yields basically ordinal information about what were the scarcest and the amplest sizes in a product group in a historic sales period. This information was utilized to change the size distributions for future deliveries by replacing one piece of the amplest size by a piece of the scarcest size in every pre-pack (this can be seen as a subgradient improvement step in an iterative size distribution heuristics based on the TDI analysis).

Empirical evidence from a blind study with twenty branches (ten of them, randomly chosen, were supplied according to TDI-based recommendations; ten of them were supplied as before) showed a significant increase in gross yield: On average, the increase in the gross yield in our blind study was around one percentage point. The probability that gross yield improvements of at least 0.25 percentage points occurred is at least 87.6 (even $95.5 \%$ if inconsistent data is repaired in a plausible way). And: this was the result of a single iteration of the optimization procedure, which did not result in perfectly balanced Top-Dog-Indices.

Given the large economies of scale of a fashion discounter, we consider the TDI a valuable contribution to revenue management tools in this business sector. Moreover, to the best of our knowledge, our blind study is the first published study that evaluates a revenue management method in the apparel retailer industry by comparing simultaneously obtained business results of test-branches (optimized) and control-branches (no action).

The draw-back of the TDI is its lack of information about the cardinal expected revenue for a given size distribution of the supply. This is partly due to the fact that the loss of a bad size distribution is closely related to the markdown policy of the discounter. This markdown policy, however, is itself subject of revenue management methods. Therefore, we regard the integration of size and price optimization, as is done in our project BFS-DISPO, as a valuable direction of further research .

\section{REFERENCES}

1. Gabriel R. Bitran, René Caldentey, and Mondscheinm Susana V., Coordinating clearance markdown sales of seasonal products in retails chains, Operations Research 46 (1998), 609-624.

2. L. M. A. Chan, Z. J. Max Shen, David Simchi-Levi, and Julie L. Swann, Coordination of pricing and inventory decisions: A survey and classification, Handbook of Quantitative Supply Chain Analysis: Modeling in the E-Business Era (David Simchi-Levi, S. D. Wu, and Z. J. Max Shen, eds.), Kluwer Academic Publishers, 2006, pp. 335-392

3. Wedad Elmaghraby and Pinar Keskinocak, Dynamic pricing in the presence of inventory considerations: Research overview, current practices, and future directions, Management Science 49 (2003), 1287-1309.

4. A. Gürhan Kök and Marshall L. Fisher, Demand estimation and assortment optimization under substitution: methodology and application, Preprint FRPS04-135, Duke University, 2006, To appear in Operations Research.

5. Iana Kouris, Dynamic pricing strategies: Markdown procedure for a textile retailer, Diplomarbeit, Universität Bayreuth, Fakultät für Mathematik, Physik und Informatik, 2007. 
6. Menachem Levanoni, Ying Tat Leung, and Sanjay Elathur Ramaswamy, Method and apparatus suitable for demand forecasting, US Patent 6976001, 2006.

7. Siddharth Mahajan and Garrett van Ryzin, Stocking retail assortments under dynamic consumer substitution, Operations Research 49 (2001), 334-351.

8. Dawn M. Rose, Steven M. Leven, and Jonathan W. Woo, Assortment decisions, US Patent 7006981, 2006.

9. Christoph Wopperer, Optimierung der Preisreduzierungsstrategie eines Textildiscounters, Diplomarbeit, Universität Bayreuth, Fakultät für Mathematik, Physik und Informatik, 2007.

Sascha Kurz, Fakultät für Mathematik, Physik und Informatik, Universität Bayreuth, Germany E-mail address: sascha.kurz@uni-bayreuth.de

Jörg Rambau, Fakultät für Mathematik, Physik und Informatik, Universität Bayreuth, Germany E-mail address: joerg.rambau@uni-bayreuth.de

Jörg Schlüchtermann, FAKultät Für Rechts- und Wirtschaftswissenschaften, Universität Bayreuth, GERMANY

E-mail address: j.schluechtermann@uni-bayreuth.de

Rainer Wolf, Betriebswirtschaftliches Forschungszentrum Für Fragen der mitTelständischen WiRtschaft E. V. An Der Universität Bayreuth, Germany

E-mail address: rainer.wolf@uni-bayreuth.de 